\title{
Analytical approaches for the electronic structure of $\mathrm{C}_{60}$
}

\author{
Yeong-Lieh Lin and Franco Nori \\ Department of Physics, University of Michigan, Ann Arbor, MI 48109-1120, USA
}

Received 23 September 1993; accepted for publication 6 October 1993

Communicated by L.J. Sham

\begin{abstract}
We use the recursion and path-integral methods to analytically study the electronic properties of a neutral $\mathrm{C}_{60}$ molecule. We obtain closed-form analytic expressions of the eigenvalues and eigenfunctions for both $\pi$ and $\sigma$ states as well as the Green functions and the local densities of states, which can be probed experimentally, around any site and around several ring clusters.
\end{abstract}

\section{Introduction}

Undoped and doped $\mathrm{C}_{60}$ molecules have recently generated enormous interest among chemists, physicists and materials scientists. In the present work, we focus on an analytical study of the electronic properties of a single neutral $\mathrm{C}_{60}$ molecule. Most studies on the electronic structure of $\mathrm{C}_{60}$ have been numerical. Here we derive closed-form expressions for the energy levels and eigenfunctions using the recursion method. The same energy levels are also obtained from a path-integral (or moment) method. This approach follows Feynman's programme: to compute physical quantities from sums over paths. All the calculations are done analytically, either by hand or with the assistance of symbolic manipulation software. These two approaches (i.e., recursion and path-integral methods) are significantly different from the ones used so far. Furthermore, they are neither numerical nor require the use of group theory. Diagonalizations are performed by iteratively applying the Hamiltonian on initial states. The beauty of the recursion method for $\mathrm{C}_{60}$ lies in the fact that the recurrence terminates very quickly (e.g., after four iterations only), providing exact and very concise expressions for the parameters of the recursion.

For a long time, the recursion and moment methods have provided a powerful numerical tool to study the electronic structure of solids. This paper focuses neither on a solid nor on a numerical method; therefore it follows a very unusual and novel approach.
Here we focus on a molecule, instead of a solid. It also turns out that our method is definitely nonstandard in molecular physics. Why solve the spectroscopy (eigenvalues and eigenfunctions) of an atom or molecule analytically, when it can be solved numerically? This question can be applied, for instance, to the hydrogen atom. Its spectroscopy is solved in textbooks using different analytical approaches, instead of simply presenting the straightforward numerical results. This is done because analytical solutions complement and enrich our quantitative and precise understanding of a problem.

To study the electronic properties of a single $C_{60}$ molecule, we consider the four carbon valence electrons $2 \mathrm{~s}, 2 \mathrm{p}_{x}, 2 \mathrm{p}_{\mathrm{y}}$, and $2 \mathrm{p}_{z}$. The $\pi$ states are formed by the sixty $2 \mathrm{p}_{z}$ orbitals, each pointing along the radial direction. A nearest-neighbor tight-binding Hamiltonian with unequal hopping integrals for single and double bonds is used to model $\pi$ states: $\hat{H}=-\sum_{\langle i j\rangle} t_{i j} c_{i}^{+} c_{j}$. We assume the hopping amplitude to be unity for every single bond and $t$ for each double bond. In addition to the radial orbital $2 \mathbf{p}_{z}$, each atom has three other orbitals, $2 \mathrm{~s}, 2 \mathrm{p}_{x}$ and $2 \mathrm{p}_{y}$, distributed on the plane tangential to the surface of the molecule. The mixture of these three orbitals at each site produces three $\mathrm{sp}^{2}$ hybrid orbitals. We now have two different kinds of hopping integrals: $V_{1}$ between orbitals on the same carbon site and $V_{2}$ between orbitals on different atoms that are associated with the same bond. The tight-binding Hamiltonian for the $\sigma$ states thus can be written as 
$\hat{H}_{\sigma}=-V_{1} \sum_{i, \alpha \neq \beta} c_{i \alpha}^{+} c_{i \beta}-V_{2} \sum_{\langle i j\rangle} c_{i \alpha}^{+} c_{j \alpha}$.

Here, $i$ denotes the carbon site and $\alpha$ the hybridized orbitals. Since the overlap integral between $\pi$ and $\sigma$ orbitals is negligible, the $\pi$-state and $\sigma$-state Hamiltonians can be treated independently. Admittedly, this is a simplified model (the "Ising model") for the electronic structure of $\mathrm{C}_{60}$ : the "hydrogen atom" of the main fullerene family of $\mathrm{C}_{60 \cdot n^{2}}$ molecules. However, its understanding is a convenient stepping stone to the study of more complex models. In this paper, we pursue several approaches to the analytical study of the spectroscopy of an important molecule. We would like to emphasize that the approaches described here are not intended to substitute more traditional methods, but to present alternative viewpoints and complementary results. The literature on $\mathrm{C}_{60}$ is vast, and here we do not attempt a review. The interested reader is referred to refs. [1-7], and references therein.

\section{Recursion method approach}

Let us begin with a brief outline of the recursion method [8] for obtaining the eigenvalues and eigenfunctions. It is important to stress that this method is almost always used numerically, while here will be implemented analytically. First, one must choose an appropriate normalized starting state $\left|f_{0}\right\rangle$. Further states are iteratively generated by the recurrence relation

$\hat{H}\left|f_{n}\right\rangle=a_{n}\left|f_{n}\right\rangle+b_{n+1}\left|f_{n+1}\right\rangle+b_{n}\left|f_{n-1}\right\rangle$,

with $b_{0}\left|f_{-1}\right\rangle \equiv 0$. Here, the real parameters $a_{n}$ and $b_{n+1}$ (chosen to be positive) are determined by $a_{n}=\left\langle f_{n}|\hat{H}| f_{n}\right\rangle$ and $b_{n+1}=\left\langle f_{n+1}|\hat{H}| f_{n}\right\rangle$. The process terminates at $\left|f_{N-1}\right\rangle$ with $b_{N}=0$. The constructed orthonormal states $\left\{\left|f_{0}\right\rangle, \ldots,\left|f_{N-1}\right\rangle\right\}$ along with the parameters $\left\{a_{0}, \ldots, a_{N-1}\right\}$ and $\left\{b_{1}, \ldots, b_{N-1}\right\}$ thus constitute the "chain model" [8] of a given Hamiltonian. This procedure essentially amounts to a change of basis. The energy levels can be achieved by constructing the following polynomials with

$$
\begin{aligned}
& P_{-1}(E)=0, \quad P_{0}(E)=1, \\
& P_{n+1}(E)=\frac{\left(E-a_{n}\right) P_{n}(E)-b_{n} P_{n-1}(E)}{b_{n+1}} .
\end{aligned}
$$

The eigenvalues $E_{\lambda}$ are determined by the $N$ zeros of the last polynomial $P_{N}(E)=0$, with an arbitrary nonzero $b_{N}$. The eigenfunctions are then

$\frac{1}{\mathscr{N}_{\lambda}} \sum_{n=0}^{N-1} P_{n}\left(E_{\lambda}\right)\left|f_{n}\right\rangle$

where

$N_{\lambda}=\left(\sum_{n=0}^{N-1} P_{n}^{2}\left(E_{\lambda}\right)\right)^{1 / 2}$

are the normalization constants.

We now come to the application of this approach to analytically study the electronic structure of a fullerene molecule, focusing first on the $\pi$ states. We will use two alternative sets (denoted by $\mathscr{A}$ and $\mathscr{B}$ ) of starting states. Each set consists of two initial states from which the same solutions for the eigenvalues and eigenfunctions can be derived. This consistency ensures the equivalent interpretation of the results from these two sets of initial states. We denote by $|j\rangle$ the $2 \mathrm{p}_{z}$ orbital centered at the $j$ th atom.

\subsection{Case A: initial states on a single ring}

The first set of initial states starts from a 5 -atom pentagon ring, $\left|u_{0}\right\rangle$, and a 6 -atom hexagon ring, $\left|v_{0}\right\rangle$. The beauty of the recursion method for $\mathrm{C}_{60}$ is that the recurrence terminates very quickly, exactly at $\left|u_{7}\right\rangle$ as well as $\left|v_{7}\right\rangle$. As a result, we obtain two groups of exact and very concise formulas for $a_{0}-a_{7}$, and $b_{1}-b_{7}$. All these states and their respective parameters are summarized in table 1.

We can then respectively construct two polynomials $P \mathrm{p}(E)$ and $P_{8}^{\mathrm{h}}(E)$ which are of eighth degree in $E$. Here the superscript $\mathrm{p}$, for pentagon, (h for hexagon ) refers to the polynomial constructed from the group of $a$ 's and $b$ 's generated by $\left|u_{0}\right\rangle\left(\left|v_{0}\right\rangle\right)$. The roots of these two polynomials can be analytically obtained and correspond to the electronic energy levels. We then obtain a total of 16 distinct eigenvalues. When $t=1$, a common root -1 exists for both polynomials $P \mathrm{~g}$ and $P_{8}^{\mathrm{h}}$. We thus have 15 distinct energy levels. It is also straightforward to obtain the eigenvectors. 
Table 1

States, $\left|u_{n}\right\rangle\left(\left|v_{n}\right\rangle\right)$, and parameters, $a_{n}$ and $b_{n+1}$, obtained by recursively applying the Hamiltonian on an initial state localized on an elementary pentagon ring $\left|u_{0}\right\rangle$ (hexagon ring $\left|v_{0}\right\rangle$ ). For $n=4,5,6$ and $7, a_{n}=a_{7-n}, b_{n}=b_{8-n}$ and $\left|u_{n}\right\rangle\left(\left|v_{n}\right\rangle\right)$ are obtained by changing the constituting orbitals in $-\left|u_{7-n}\right\rangle\left(\left|v_{7-n}\right\rangle\right)$ into the antipode ones. The labelling is as follows. Starting from a pentagon ring, $j$ runs from 1 to 5 . For the second shell (one bond away from this pentagon), $j$ runs from 6 to 10 . For the third (fourth) shell, which is one bond away from the second (third) shell, $j$ runs from 11 to 20 (21 to 30). For concreteness, site $6(11)$ is connected to 1,11 and 12 (6, 20 and 21 ). These 30 atoms occupy one hemisphere. The 30 atoms on the other hemisphere are denoted by $1^{\prime}$ through $30^{\prime}$. Note that $j$ and $j^{\prime}$ are antipodes, i.e., related by inversion along the molecule center. Starting from a hexagon ring, we have similar labellings, except that site $7(21)$ is connected to 1,13 and $21(7,20$ and 30$)$.

\begin{tabular}{ccccccc}
\hline$n$ & $\left|u_{n}\right\rangle$ & $a_{n}$ & $b_{n+1}$ & $\left|v_{n}\right\rangle$ & $a_{n}$ & $b_{n+1}$ \\
\hline 0 & $\frac{1}{\sqrt{5}} \sum_{j=1}^{5}|j\rangle$ & -2 & $t$ & $\frac{1}{\sqrt{6}} \sum_{j=1}^{6}(-1)^{j+1}|j\rangle$ & $1+t$ & 1 \\
1 & $-\frac{1}{\sqrt{5}} \sum_{j=6}^{10}|j\rangle$ & 0 & $\sqrt{2}$ & $\frac{1}{\sqrt{6}} \sum_{j=7}^{12}(-1)^{j}|j\rangle$ & 0 & $t$ \\
2 & $\frac{1}{\sqrt{10}} \sum_{j=11}^{20}|j\rangle$ & $-t$ & 1 & $\frac{1}{\sqrt{6}} \sum_{j=14}^{15}(-1)^{j+1}(|j\rangle+|j+3\rangle+|j+6\rangle)$ & 1 & 1 \\
3 & $-\frac{1}{\sqrt{10}} \sum_{j=21}^{30}|j\rangle$ & -1 & $t$ & $\frac{1}{\sqrt{6}} \sum_{j=23}^{24}(-1)^{j+1}(|j\rangle+|j+3\rangle+|j+6\rangle)$ & 0 & $t$ \\
\hline
\end{tabular}

\subsection{Case $\not 2$ : initial states on two antipode rings}

In this approach, we exploit the symmetry property that the inversion operator leaves the $\mathrm{C}_{60} \mathrm{~mol}-$ ecule invariant. We therefore take the first (second) starting state $\left|\phi_{0}\right\rangle\left(\left|\varphi_{0}\right\rangle\right)$ as a linear combination of the orbitals on two opposite (i.e., antipodes) pentagon (hexagon) rings. Following the same procedure, we find the very convenient and remarkable result that the recurrence terminates even faster at $\left|\phi_{3}\right\rangle\left(\left|\varphi_{3}\right\rangle\right)$. The parameters generated from $\left|\phi_{n}\right\rangle$ are $a_{0}=-2, a_{1}=0, a_{2}=-t, a_{3}=-1-\mathscr{P} t, b_{1}=t$, $b_{2}=\sqrt{2}$ and $b_{3}=1$. Also from $\left|\varphi_{n}\right\rangle$ we obtain $a_{0}=1+t, a_{1}=0, a_{2}=1, a_{3}=\mathscr{P} t, b_{1}=1, b_{2}=t$ and $b_{3}=1$. Here $\mathscr{P}$ stands for parity. Due to the two possible values of the parity, we can construct four polynomials $P_{4}^{\mathrm{p}+}(E), P_{4}^{\mathrm{p}-}(E), P_{4}^{\mathrm{h}+}(E)$ and $P_{4}^{\mathrm{h}}-(E)$. Each one is fourth degree in $E$. The superscript $\mathrm{p}+$ stands for the polynomial constructed from $\left|\phi_{0}\right\rangle$ with even parity, and similarly for the others. By analytically solving these four polynomials, we obtain the same 16 eigenvalues obtained above (case $\mathscr{A}$ ). Similarly, we can obtain the eigenvectors which are also equal to those from the alternative $\mathscr{A}$.

\subsection{Summary of results}

In table 2, we summarize the eigenvalues and the corresponding eigenvectors for the case $t=1$. In table
3 , we present the closed-form eigenvalues explicitly expressed in terms of the single-bond and doublebond hopping integrals $t_{1}$ and $t_{2}$. Since the other degenerate eigenvectors can be generated by standard group theory analysis, we therefore only present the eigenfunctions derived from the pure application of the recursion method. Starting from an initial state localized on one, $\left|u_{0}\right\rangle$, or two, $\left|\phi_{0}\right\rangle$, pentagons we generate most of the low energy levels because every pentagon has single bonds. On the other hand, since every hexagon has double bonds and more overall bonds, iterations on $\left|v_{0}\right\rangle$ or $\left|\varphi_{0}\right\rangle$ produce most of the high energy levels.

\subsection{Relations between alternatives $\mathscr{A}$ and $\mathscr{B}$}

It is worthwhile to point out the following relations between the cases $\mathscr{A}$ and $\mathscr{B}$ presented before. First, roots solved from $P \mathrm{~g}(E)\left(P_{8}^{\mathrm{h}}(E)\right)$ are identical to those from $P_{4}^{\mathrm{p}+}(E)$ and $P_{4}^{\mathrm{p}}-(E)\left(P_{4}^{\mathrm{h}+}(E)\right.$ and $\left.P_{4}^{\mathrm{h}}-(E)\right)$. Second, for $n=0,1,2$ and 3, $\left|\phi_{n}\right\rangle=\left(\left|u_{n}\right\rangle-\mathscr{P}\left|u_{7-n}\right\rangle\right) / \sqrt{2}$, and $\left|\varphi_{n}\right\rangle=\left(\left|v_{n}\right\rangle\right.$ $\left.+\mathscr{P}\left|v_{7-n}\right\rangle\right) / \sqrt{2}$. Third, for those eigenvalues $E_{\lambda}$ which are common roots of $P_{8}^{\mathrm{p}}$ and $P_{4}^{\mathrm{p}} \pm$ (or $P_{8}^{\mathrm{h}}$ and $\left.P_{4}^{\mathrm{h} F}\right), P_{7-n}\left(E_{\lambda}\right)=\mp P_{n}\left(E_{\lambda}\right)$. Here, all the $P_{n}\left(E_{\lambda}\right)$ 's refer to the polynomials constructed in the case $\mathscr{A}$ and $n=0,1,2,3$. Fourth, $P_{1}\left(E_{\lambda}\right), P_{2}\left(E_{\lambda}\right)$ and $P_{3}\left(E_{\lambda}\right)$ calculated from the two alternatives $\mathscr{A}$ and $\mathscr{B}$ are the same. Fifth, for the same eigenvector, the normali- 
Table 2

The eigenvalues $E_{\lambda}$ and the corresponding eigenvectors $\left(1 / \mathcal{N}_{\lambda}\right) \sum_{n=0}^{3} P_{n}\left(E_{\lambda}\right)\left|f_{n}\right\rangle$ for $\mathrm{C}_{60}$ with $t=1$. Here $\left|\phi_{n}^{ \pm}\right\rangle\left(\left|\varphi_{n}^{ \pm}\right\rangle\right)$denotes $\left|\phi_{n}\right\rangle$ $\left(\left|\varphi_{n}\right\rangle\right)$ with $\mathscr{P}= \pm 1$. Here $\alpha=[2(19+\sqrt{5})]^{1 / 2}$ and $\beta=[2(19-\sqrt{5})]^{1 / 2}$.

\begin{tabular}{|c|c|c|c|c|c|}
\hline$E_{\lambda}$ & $P_{1}\left(E_{\lambda}\right)$ & $P_{2}\left(E_{\lambda}\right)$ & $P_{3}\left(E_{\lambda}\right)$ & $\mathcal{N}_{\lambda}$ & $\left|f_{n}\right\rangle$ \\
\hline-3 & -1 & $\sqrt{2}$ & $-\sqrt{2}$ & 6 & $\left|\phi_{n}^{+}\right\rangle$ \\
\hline-1 & 1 & $-\sqrt{2}$ & $-\sqrt{2}$ & 6 & $\left|\phi_{n}^{+}\right\rangle$ \\
\hline$\frac{1}{2}(-1 \pm \sqrt{13})$ & $\frac{1}{2}(3 \pm \sqrt{13})$ & $\frac{1}{4} \sqrt{2}(3 \pm \sqrt{13})$ & $\frac{1}{2} \sqrt{2}$ & $\frac{1}{4}(39 \pm 9 \sqrt{13})$ & $\left|\phi_{n}^{+}\right\rangle$ \\
\hline$\frac{1}{4}(-1+\sqrt{5} \pm \alpha)$ & $\frac{1}{4}(5+\sqrt{5} \pm \alpha)$ & $\frac{1}{16} \sqrt{2}[6+2 \sqrt{5} \pm(\sqrt{5}+1) \alpha]$ & $\frac{1}{16} \sqrt{2}[-4+8 \sqrt{5} \pm(\sqrt{5}-1) \alpha]$ & $\frac{1}{8}(95+5 \sqrt{5}) \pm \frac{1}{16}(25+\sqrt{5}) \alpha$ & $\left|\phi_{n}^{-}\right\rangle$ \\
\hline$\frac{1}{4}(-3-\sqrt{5} \pm \beta)$ & $\frac{1}{4}(5-\sqrt{5} \pm \beta)$ & $\frac{1}{16} \sqrt{2}[6-2 \sqrt{5} \mp(\sqrt{5}-1) \beta]$ & $\frac{1}{16} \sqrt{2}[-4-8 \sqrt{5} \mp(\sqrt{5}+1) \beta]$ & $\frac{1}{8}(95-5 \sqrt{5}) \pm \frac{1}{16}(25-\sqrt{5}) \beta$ & $\left|\phi_{n}^{-}\right\rangle$ \\
\hline 2 & 0 & -1 & -1 & 3 & $\left|\varphi_{n}^{+}\right\rangle$ \\
\hline-1 & -3 & 2 & -1 & 15 & $\left|\varphi_{n}^{+}\right\rangle$ \\
\hline$\frac{1}{2}(3 \pm \sqrt{5})$ & $\frac{1}{2}(-1 \pm \sqrt{5})$ & $\frac{1}{2}(-1 \pm \sqrt{5})$ & $\frac{1}{2}(3 \mp \sqrt{5})$ & $\frac{1}{2}(15 \mp 5 \sqrt{5})$ & $\left|\varphi_{n}^{+}\right\rangle$ \\
\hline$\frac{1}{2}(1 \pm \sqrt{5})$ & $\frac{1}{2}(-3 \pm \sqrt{5})$ & $\frac{1}{2}(-1 \mp \sqrt{5})$ & $\frac{1}{2}(1 \mp \sqrt{5})$ & $\frac{1}{2}(15 \mp 3 \sqrt{5})$ & $\left|\varphi_{n}^{-}\right\rangle$ \\
\hline$\frac{1}{2}(1 \pm \sqrt{17})$ & $\frac{1}{2}(-3 \pm \sqrt{17})$ & $\frac{1}{2}(5 \mp \sqrt{17})$ & $-4 \pm \sqrt{17}$ & $51 \mp 12 \sqrt{17}$ & $\left|\varphi_{n}^{-}\right\rangle$ \\
\hline
\end{tabular}

\section{Table 3}

The eigenvalues and the corresponding degree of degeneracy (either for the plus or minus sign) for $\mathrm{C}_{60}$. The characteristic polynomials from which those eigenvalues are solved are indicated in the first column. Here $\tau=\left\{2\left[8 t_{2}^{2}-(4+4 \sqrt{5}) t_{2} t_{1}\right.\right.$ $\left.\left.+(15+5 \sqrt{5}) t_{1}^{2}\right]\right\}^{1 / 2}$ and $\gamma=\left\{2\left[8 t_{2}^{2}-(4-4 \sqrt{5}) t_{2} t_{1}+\right.\right.$ $\left.\left.(15-5 \sqrt{5}) t_{1}^{2}\right]\right\}^{1 / 2}$. Also $\eta$ and $\xi$ satisfy $\frac{1}{54}\left(16 t_{2}^{3}-24 t_{2}^{2} t_{1}+\right.$ $\left.12 t_{2} t_{1}^{2}+25 t_{1}^{3}\right)=\eta^{3}-3 \eta \xi^{2}$ and $\frac{1}{18}\left[3\left(64 t_{2}^{4}-160 t_{2}^{3} t_{1}+288 t_{2}^{2} t_{1}^{2}\right.\right.$ $\left.\left.-200 t_{2} t_{1}^{3}+125 t_{1}^{4}\right)\right]=3 \eta^{2} \xi-\xi^{3}$.

\begin{tabular}{lcl}
\hline & Energy & Degeneracy \\
\hline$P_{4}^{p^{+}}$ & $-\left(2 t_{1}+t_{2}\right)$ & 1 \\
& $-\frac{1}{3}\left(t_{1}+t_{2}\right)+2 \eta$ & 5 \\
& $-\frac{1}{3}\left(t_{1}+t_{2}\right)-\eta \pm \sqrt{3} \xi$ & 5 \\
$P_{4}^{p^{-}}$ & $\frac{1}{4}\left[(-3+\sqrt{5}) t_{1} \pm \tau\right]$ & 3 \\
& $\frac{1}{4}\left[(-3-\sqrt{5}) t_{1} \pm \gamma\right]$ & 3 \\
$P_{4}^{\mathrm{h}+}$ & $\frac{1}{2}\left(t_{1} \pm \sqrt{5 t_{1}^{2}+4 t_{2}^{2}}\right)$ & 4 \\
& $t_{2}+\frac{1}{2}(1 \pm \sqrt{5}) t_{1}$ & 3 \\
$P_{4}^{\mathrm{h}-}$ & $\frac{1}{2}\left(t_{1} \pm \sqrt{5 t_{1}^{2}-4 t_{1} t_{2}+4 t_{2}^{2}}\right)$ & 5 \\
& $\frac{1}{2}\left(t_{1} \pm \sqrt{5 t_{1}^{2}+8 t_{1} t_{2}+4 t_{2}^{2}}\right)$ & 4 \\
\hline
\end{tabular}

zation constants $\mathscr{N}_{\mathscr{A}}$ and $\mathscr{N}_{\mathscr{A}}$, calculated in $\mathscr{A}$ and $\mathscr{B}$ respectively, satisfy $\mathscr{N}_{\mathscr{A}}=\sqrt{2} \mathcal{N}_{\mathscr{A}}$. From the above five properties, the equivalence of results from both alternatives becomes clear. In fact, from the results of alternative $\mathscr{A}$, we can understand the parity property associated with $\mathrm{C}_{60}$.

\section{Path-integral (or moment) approach}

In this method, the central task is the computation of path-integrals (moments), defined by $\mathscr{M}_{l} \equiv$ $\left\langle j\left|\hat{H}^{l}\right| j\right\rangle$, where the order, $l$, is a positive integer. The physical meaning of $\mathscr{M}_{l}$ is as follows. The Hamiltonian $\hat{H}$ is applied $l$ times to an initial $2 \mathrm{p}_{z}$ state $|j\rangle$. Each time $\hat{H}$ is applied, the electron gains a certain amount of kinetic energy. This enables the electron to hop through $l$ bonds, reaching the final state $A^{l}|j\rangle$. The path-integral just equals the total kinetic energy gained by the electron returning to the starting site $j$ after hopping $l$ steps. It is obvious that $\mathscr{M}_{l}$ will be zero when the $l$-hops path does not return to the initial site. For the case $t=1$, the absolute value of the moment $\mathscr{M}_{l}$ is the total number of closed paths of $l$ steps starting and ending at the same site.

Below we describe two approaches for analytically calculating the path-integrals on a $\mathrm{C}_{60}$ molecule. Let the orbital $|1\rangle$ be our starting state. Note that the action of the Hamiltonian on an arbitrary state results in three nearest-neighbor states with an additional factor accounting for the respective bond hopping energy. Our strategy is: each time the power of the Hamiltonian increases by one, we move to the 
nearest-neighboring sites. Also the factor on each site is the sum of the factors on the three nearest-neighbor sites times the bond hopping integral between sites. From its definition, it is evident that the moment of order $l$ is just the factor on the site 1 for $\hat{H}^{l}$. We can thus generate all the moments to any order one by one. Because of the mirror-symmetry of $\mathrm{C}_{60}$, we need only concern ourselves with the sites in one of the two hemispheres. Furthermore, because of the geometrical equivalence, with respect to site 1 , of eight pairs of sites, the total number of independent sites can be reduced to 24 .

An alternative procedure can be implemented by using symbolic manipulation software on a computer. Define an auxiliary quantity, $W_{l}(j)$, which is the sum over all possible paths of $l$ steps on which an electron may hop from site 1 to $j$. From the connectivity of $\mathrm{C}_{60}$, we can then construct 24 independent recurrence relations like $W_{l+1}(k)=$ $-t W_{l}(p)-W_{l}(q)-W_{l}(r)$. The recurrence relation states that the site $k$ can be reached by taking the $(l+1)$ th step from the nearest-neighbors $p, q$ and $r$. The factors $-t$ and -1 account for the connecting bond-hopping integral. With the conditions $W_{0}(1)=1$ and $W_{0}(j)=0$ for the rest of $j$ s, we can obtain the moments to any order as $\mathscr{M}_{l}=W_{l}(1)$. The correctness of the calculated moments is assured by the consistency of the results from these two approaches. It is evident that through these two approaches we can also obtain the quantities $\left\langle 1\left|\hat{H}^{l}\right| j\right\rangle$ for $j \neq 1$ which can be appropriately interpreted as the "sum-over-paths" between sites 1 and $j$. For instance, $\left\langle 1\left|\hat{H}^{\prime}\right| j\right\rangle$ just equals $W_{l}(j)$.

To obtain the energy spectrum, we need to express the parameters $a_{n}$ and $b_{n+1}$ in terms of the moments. We employ the following formulae [9]: define the matrix $M$ with the first row elements $M_{0, l} \equiv \mathscr{M}_{l}$. The other rows are evaluated by

$$
\begin{aligned}
M_{n, l} & =\frac{M_{n-1, l+2}-M_{n-1,1} M_{n-1, l+1}}{M_{n-1,2}-M_{n-1,1}^{2}} \\
& -\sum_{k=0}^{l-1} M_{n, k} M_{n-1, l-k},
\end{aligned}
$$

here $n \geqslant 1$ and $l=0,1, \ldots$. The $a_{n}$ 's and $b_{n+1}$ 's are obtained from $a_{n}=M_{n, 1}$ and $b_{n+1}^{2}=M_{n, 2}-M_{n, 1}^{2}$, for $n=0,1,2, \ldots$. We analytically find that $b_{15}^{2}$ exactly equals 0 for $t=1$ and $b_{16}^{2}$ exactly equals 0 for arbi- trary $t$. For the case $t=1$, it turns out that the highest order moment we need is $l=30$. Through the calculated parameters, we can construct the polynomial $P_{15}(E)$. We thus obtain 15 energy levels. The results are identical to those obtained from the recursion method approaches. Similar conclusions can also be drawn for $t \neq 1$.

\section{Alternative application of the path-integral method}

It is worthwhile to incorporate the inversion symmetry property in the path-integral approach in order to obtain the results in a faster and much compact manner. Therefore, instead of focusing on a single localized state, we turn to the computation of the moments with respect to the state $\left|I_{ \pm}\right\rangle=$ $\left(|1\rangle \pm\left|1^{\prime}\right\rangle\right) / \sqrt{2}$, where atoms 1 and $1^{\prime}$ are antipodes. It is a simple exercise to construct the following identity $\left\langle I_{ \pm}\left|\hat{H}^{\prime}\right| I_{ \pm}\right\rangle=\left\langle 1\left|\hat{H}^{\prime}\right| 1\right\rangle \pm\left\langle 1\left|\hat{H}^{\prime}\right| 1^{\prime}\right\rangle$. The moments $\left\langle I_{ \pm}\left|\hat{H}^{\prime}\right| I_{ \pm}\right\rangle$can then be readily obtained since the quantities $\left\langle 1\left|\hat{H}^{l}\right| 1\right\rangle$ and $\left\langle 1\left|\hat{H}^{l}\right| 1^{\prime}\right\rangle$ are already available.

For the case $t=1$, the highest order we need for $\left\langle I_{+}\left|\hat{H}^{\prime}\right| I_{+}\right\rangle\left(\left\langle I_{-}\left|\hat{H}^{l}\right| I_{-}\right\rangle\right)$is $14(16)$, because $b_{7}^{2}\left(b_{8}^{2}\right)$ calculated from these moments turns out to be exactly 0 . We thus analytically obtain 7 (8) eigenvalues which are identical to those belonging to the $\mathscr{P}=+1(\mathscr{P}=-1)$ category from the recursion method. For an arbitrary $t$, we need moments $\left\langle I_{ \pm}\left|\hat{H}^{\prime}\right| I_{ \pm}\right\rangle$up to order 16 . We analytically find that $b_{8}^{2}$ exactly equals 0 in both cases. Consequently, consistent results for the eigenvalues are recovered. In this section, we have presented an unconventional choice of initial states and concentrated on the moments with respect to these states. This approach is even more efficient (than the one described in the previous section) in analytically obtaining the energy eigenvalues.

\section{Relationship between the recursion and path- integral methods}

Generally speaking, the path-integral method is closely related to the recursion method, especially in 
the aspect that both approaches lead to the same results for the parameters $a_{n}$ and $b_{n+1}^{2}$. In this section, we illuminate this point by showing that the same expressions for the parameters in the recursion method case $\mathscr{B}$ can be obtained through the moment method. As the initial states are $\left|\phi_{0}\right\rangle$ and $\left|\varphi_{0}\right\rangle$ in this case, the moments we now need to compute are $\left\langle\phi_{0}\left|\hat{H}^{\prime}\right| \phi_{0}\right\rangle$ and $\left\langle\varphi_{0}\left|\hat{H}^{l}\right| \varphi_{0}\right\rangle$. It is straightforward to obtain these moments through the techniques previously discussed.

Anticipating the termination at $b_{4}^{2}$, we only need these moments up to order 8 . We thus obtain $a_{n}$ 's and $b_{n+1}^{2}$ 's which are consistent with those derived directly from the recursion method. We thus demonstrate the fact that the same results for the parameters $a_{n}$ and $b_{n+1}^{2}$ can be obtained by using either the recursion or moment methods. The advantage of the recursion method is that we can simultaneously generate the states and the parameters. However, it is sometimes difficult to derive the states and parameters when the recursion method is applied to some starting state, while the moment method provides standard procedures to calculate the parameters after the moments are obtained.

\section{Local density of states}

In the recursion and path-integral methods, the diagonal element of the Green function $(E-\hat{H})^{-1}$ can be expressed as a continued fraction $[8,9]$,

$G_{0}(E)=\left\langle f_{0}\left|\frac{1}{E-\hat{H}}\right| f_{0}\right\rangle$

$=1 /\left(E-a_{0}-b_{0}^{2} /\left(E-a_{1}-\ldots-b_{N-1}^{2} /\left(E-a_{N-1}\right) \ldots\right)\right)$.

The local density of states (LDOS) $\rho(E)$ for $\left|f_{0}\right\rangle$ is related to the imaginary part of $G_{0}(E)$ by

$\rho(E)=\lim _{\epsilon \rightarrow 0}\left(-\frac{1}{\pi} \operatorname{Im} G_{0}(E+\mathrm{i} \epsilon)\right)$.

By plugging in their respective parameters from these methods, we thus obtain the LDOS on several initial states. In principle, they are experimentally accessible by using a scanning tunneling microscope (STM) [10]. Choosing the initial state as a $2 \mathrm{p}_{z}$ orbital on a carbon atom, the path-integral method provides a local density of states and the degree of degeneracy for each energy level (since the total number of $\pi$ electrons is 60 ).

\section{Solution for the electronic $\sigma$ states}

The eigenvalues and eigenvectors of the $\sigma$-states Hamiltonian $\hat{H}_{\sigma}$ can be obtained after an analytic transformation of this Hamiltonian into a simpler one with a single hopping parameter between sites [11]. The details of this mapping and other results related to this paper will be discussed elsewhere. As a result, among the $90 \sigma$ bonding ( $90 \sigma$ antibonding) states, 30 states are lumped together at the energy level $V_{1}-V_{2}\left(V_{1}+V_{2}\right)$. The eigenvalues of the other 60 bonding (with plus sign) and 60 antibonding states (with minus sign) are

$-\frac{1}{2} V_{1} \pm V_{2} \sqrt{1+\left(V_{1} / V_{2}\right) E_{\lambda}+\frac{9}{4} V_{1}^{2} / V_{2}^{2}}$,

where $E_{\lambda}$ are the $\pi$ state eigenvalues listed in table 2 .

\section{Conclusions}

In conclusion, we use several approaches based on the recursion and path-integral methods in order to analytically study the spectroscopy of the $\mathrm{C}_{60} \mathrm{~mol}-$ ecule. In particular, we obtain closed-form analytic expressions of the eigenvalues and eigenfunctions for both $\pi$ and $\sigma$ states as well as the LDOS around any site and around several ring clusters. The former, for instance, can be experimentally measured by using STM spectroscopic techniques (see, e.g., review [10]).

\section{Acknowledgement}

We gratefully acknowledge stimulating and useful discussions with C.N. Yang and F. Guinea. FN acknowledges partial support from a GE fellowship, the NSF through grant DMR-90-01502, and SUN Microsystems.

\section{References}

[1] E. Manousakis, Phys. Rev. B 44 (1991) 10991; 48 (1993) 2024 (E). 
[2] D. Tománek and M.A. Schluter, Phys. Rev. Lett. 67 (1991) 2331.

[3] Y. Deng and C.N. Yang, Phys. Lett. A 170 (1992) 116.

[4] R. Friedberg, T.D. Lee and H.C. Ren, Phys. Rev. B 46 (1992) 14150.

[5] J. González, F. Guinea and M.A.H. Vozmediano, Phys. Rev. Lett. 69 (1992) 172; Nuclear Phys., in press; F. Guinea, J. González and M.A.H. Vozmediano, Phys. Rev. B 47 (1993) 16576.

[6] S. Satpathy, Chem. Phys. Lett. 130 (1986) 545; S. Satpathy et al., Phys. Rev. B 46 (1992) 1773.

[7] V. Elser and R.C. Haddon, Phys. Rev. A 36 (1987) 4579; Nature 325 (1987) 792.
[8] R. Haydock, in: Solid state physics, Vol. 35, eds. H. Ehrenreich, F. Seitz and D. Turnbull (Academic Press, New York, 1980).

[9] G. Grosso and G. Pastori Parravicini, in: Advances in chemical physics, Vol. 62. Memory function approaches to stochastic problems in condensed matter, eds. M.W. Evans, P. Grigolin and G. Pastori Parravicini (Wiley, New York, 1985).

[10] J.H. Weaver, Acc. Chem. Res. 25 (1992) 143, and references therein.

[11] D. Weaire and M.F. Thorpe, in: Computational methods for large molecules and localized states, eds. F. Herman, A.D. McLean and R.K. Nesbet (Plenum, New York, 1973). 